\title{
Clinical progression and familial occurrence of cerebral cavernous angiomas: the role of angiogenic and growth factors
}

\author{
Francesco Maiuri, M.D., Paolo Cappabianca, M.D., Michelangelo Gangemi, M.D., \\ Marialaura Del Basso De Caro, Ph.D., Felice Esposito, M.D., Guido Pettinato, M.D., \\ Oreste de Divitis, M.D., Chiara Mignogna, M.D., Viviana Strazzullo, Ph.D., \\ AND ENRICO DE DivitiIs, M.D.
}

Division of Neurosurgery, Department of Neurological Sciences; and Department of

Biomorphological and Functional Sciences, Section of Pathology,

Università degli Studi di Napoli Federico II, Naples, Italy

\begin{abstract}
Object. The authors studied the expression of angiogenic and growth factors and various proliferative indices in cavernous angiomas of the brain. The goal was to define whether the often progressive clinical course of both sporadic and familial forms of the lesion is correlated with different expression of these factors.

Methods. Forty-three cavernomas of the brain were investigated with immunohistochemical studies and stained for four growth factors (vascular endothelial growth factor [VEGF], tenascin, transforming growth factor- $\beta$ [TGF $\beta$ ], and platelet-derived growth factor [PDGF]), and for Ki-67 and bcl-2. The intensity of expression was tested in all cases in the walls of cavernoma vessels, in the perivascular tissue, and in the perilesional brain parenchyma. Among the 43 cavernomas, 32 were stable and sporadic single lesions less than $2 \mathrm{~cm}$ in size, whereas 11 were cavernomas larger than 2 $\mathrm{cm}$ (up to $6 \mathrm{~cm}$ ). These larger cavernomas had more aggressive behavior (documented growth in five cases, mass effect in eight, significant hemorrhage in four), familial occurrence (six cases), and/or multiple lesions (five cases).

The expression of VEGF, tenascin, and PDGF in cavernomas did not significantly differ in the two groups of patients, whereas TGF $\beta$ expression was higher in the more aggressive forms of cavernomas. The expression of Ki-67 and bcl-2 was always absent in stable lesions, and it was positive in eight (72.7\%) of 11 aggressive lesions. The perilesional brain parenchyma showed a significantly higher expression of TGF $\beta$, PDGF, and tenascin in more aggressive cavernomas.

Conclusions. The familial occurrence and more aggressive clinical behavior of cavernous angiomas of the brain are associated with higher expression of $\mathrm{Ki}-67$ and bcl-2 in the cavernoma tissue, as in other proliferative lesions. These features are also associated with higher expression of some growth factors (excluding VEGF) in the perilesional brain parenchyma, suggesting that the neighboring vasculature and glia may be predisposed to and recruited for further growth and progression.
\end{abstract}

KEY WORDS • cavernous angioma • familial lesion • growth factor $\bullet$ Ki-67 • bcl-2

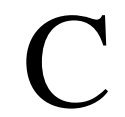

AVERNOUS angiomas of the brain are common lesions occurring with a prevalence of approximately $0.5 \% .^{35}$ Their diagnosis has been drastically improved by the widespread use of MR imaging. Familial forms with an autosomal-dominant transmission are observed in 25 to $54 \%$ of cases. . $^{12,33,47}$

Most cavernomas are indolent and stable lesions that are discovered incidentally or because of sporadic sei-

Abbreviations used in this paper: $\mathrm{CT}=$ computed tomography; $\mathrm{ECM}=$ extracellular matrix; $\mathrm{MR}=$ magnetic resonance; PCNA = proliferating cell nuclear antigen; PDGF = platelet-derived growth factor; TGF = transforming growth factor; VEGF = vascular endothelial growth factor. zures, and they remain unchanged on the follow-up studies obtained over the years. On the other hand, some cases show a more aggressive clinical behavior, which may lead to functional and even life-threatening risks. The main factors that can make a cavernoma an aggressive lesion include hemorrhage (particularly extralesional and recurrent), enlargement of the cavernomatous matrix, de novo appearance, extensive calcifications, and familial occurrence. ${ }^{15}$

Several studies ${ }^{18,37,42,45,48}$ have shown the expression of growth factors, mainly VEGF and TGF $\alpha$, in brain cavernomas. It has been suggested as well that some potential regulatory effectors of angiogenesis, mainly VEGF, may play a role in the progressive and dynamic behavior of cavernomas.

In this study, we investigated the expression of some 
growth factors and proliferative indices, including VEGF, tenascin, TGF $\beta$, PDGF, Ki-67, and bcl-2 in a series of brain cavernomas. Our aim was to define whether the indolent or progressive clinical behavior of both sporadic and familial forms is related to different expression of growth factors and proliferative indices in both cavernoma tissue and surrounding brain parenchyma.

\section{Clinical Material and Methods}

\section{Patient Selection}

We studied 43 brain cavernomas from the surgical database of cerebral vascular malformations resected in patients treated in our neurosurgical department between 1989 and 2002, with a minimum follow-up duration of 36 months. The study includes children and adults with sporadic or familial forms of cavernomas. An MR image was obtained and histological studies were performed in all patients.

\section{Demographic Data}

Of the 43 patients, 17 were male and 26 were female, and their ages ranged from 2 to 65 years (mean 31.6 years). Among them, $38(88.4 \%)$ were adults and five $(11.6 \%)$ were children younger than 15 years.

Factors that we statistically analyzed include the patient's age and sex, location and size of the cavernoma, clinical onset, familial occurrence, symptomatic hemorrhage, radiological evidence of enlargement of the cavernoma and mass effect, and de novo appearance.

To define whether the growth factors and proliferative indices may play a role in the familial occurrence and clinical course and progression of cavernomas, we have divided the 43 patients into two groups. Group I included 32 patients with indolent and stable lesions that exhibited the following features: sporadic occurrence, size less than 2 $\mathrm{cm}$, absence of mass effect, and extralesional hemorrhage. As seen in Table 1, Group II included 11 patients with cavernomas of $2 \mathrm{~cm}$ or more in size that exhibited one or more features suggesting a more aggressive clinical behavior. These included familial occurrence (six cases), documented growth (five cases, Fig. 1), mass effect (eight cases, Fig. 2), multiple lesions (five cases), and significant extralesional symptomatic hemorrhage (four cases).

\section{Tumor Specimens}

The histological sections were reviewed by two different pathologists (M.D.B.D.C. and C.M.), who were unaware of the clinicoradiological features of the tumors. Paraffin-embedded specimens were cut to obtain 5- $\mu \mathrm{m}$ sections. These were dried in a stove at $60^{\circ} \mathrm{C}$ for 24 hours; passed through a series of xylol, alcohol, and distilled water; and then processed in $3 \% \mathrm{H}_{2} \mathrm{O}_{2}$ for 10 minutes. The sections were placed in a citrate tampon in the microwave at $650 \mathrm{~W}$ (three cycles for 5 minutes) for antigen unmasking. Finally, they were incubated in a humid chamber with the primary antibody.

\section{Immunohistochemical Studies}

The immunohistochemical studies were performed for each specimen in samples obtained in the vessel walls (endothelium and subendothelium), the perivascular tissue, and the brain parenchyma surrounding the cavernoma (Fig. 3). The expression of four growth factors was tested; these included VEGF (NeoMarkers; Stratech Scientific, Ltd., Soham, Cambridgeshire, United Kingdom; 1:50 overnight), tenascin (Dako Corp., Glostrup, Denmark; 1:200 for 30 minutes), TGF $\beta$ (Santa Cruz Biotechnology, Inc., Heidelberg, Germany; 1:300 for 40 minutes), and PDGF (Santa Cruz Biotechnology, Inc.; 1:300 for 40 minutes). Specimens of skin for PDGF, breast for VEGF, gut for TGF $\beta$, and connective tissue for tenascin were used as positive controls. The negative control was obtained by eliminating the primary monoclonal antibody. The sections were then washed in Tris buffer to eliminate the excess of antibody, incubated in a humid chamber with the secondary antibody for 20 minutes, and washed again in Tris for 5 minutes. For antigen detection, alkaline phosphatase was used for tenascin, and the streptavidin-biotin complex and diaminobenzidine were used for the other growth factors. The sections were then washed in water and stained with hematoxylin for 1 minute to display the nuclei. In this way, definitive slides were prepared for microscopy.

The presence of Ki-67 was determined by using the monoclonal antibody MIB-1 (Immunotech, Inc., Westbrook, ME; dilution 1:100, overnight incubation). The streptavidin-biotin system and the diaminobenzidine were used for antigen visualization. The proliferative compo-

TABLE 1

Epidemiological features in 11 patients with aggressive cavernous angiomas*

\begin{tabular}{cclcccccc}
\hline \hline $\begin{array}{c}\text { Case } \\
\text { No. }\end{array}$ & $\begin{array}{c}\text { Age (yrs), } \\
\text { Sex }\end{array}$ & $\begin{array}{c}\text { Lesion } \\
\text { Location }\end{array}$ & $\begin{array}{c}\text { Familial } \\
\text { Size }(\mathrm{cm})\end{array}$ & $\begin{array}{c}\text { Multiple } \\
\text { Lesions }\end{array}$ & $\begin{array}{c}\text { Documented } \\
\text { Lesions }\end{array}$ & $\begin{array}{c}\text { Mass } \\
\text { Growth }\end{array}$ & $\begin{array}{c}\text { Extralesional } \\
\text { Effect }\end{array}$ & $\begin{array}{c}\text { Hemor- } \\
\text { rhage }\end{array}$ \\
\hline 1 & $12, \mathrm{~F}$ & rt temporal & 3.5 & + & - & + & + & + \\
2 & $58, \mathrm{M}$ & lt frontal & 2.5 & + & + & - & - & - \\
3 & $50, \mathrm{M}$ & rt frontal & 3 & + & - & - & + & - \\
4 & $12, \mathrm{~F}$ & lt temporal & 3 & + & - & - & + & - \\
5 & $15, \mathrm{~F}$ & rt frontotemporal & 3 & + & + & - & + & + \\
6 & $28, \mathrm{~F}$ & rt frontal & 2.5 & + & - & - & - & - \\
7 & $40, \mathrm{M}$ & lt parietal & 6 & - & - & + & + & + \\
8 & $2, \mathrm{~F}$ & rt frontal & 5 & - & + & + & + & - \\
9 & $3, \mathrm{~F}$ & brainstem & 2.5 & - & + & + & + & - \\
10 & $23, \mathrm{M}$ & rt frontal & 2.5 & - & - & + & + & + \\
11 & $40, \mathrm{~F}$ & lt frontal & 2.5 & - & + & - & - & - \\
\hline
\end{tabular}

$*+=$ present; $-=$ absent. 

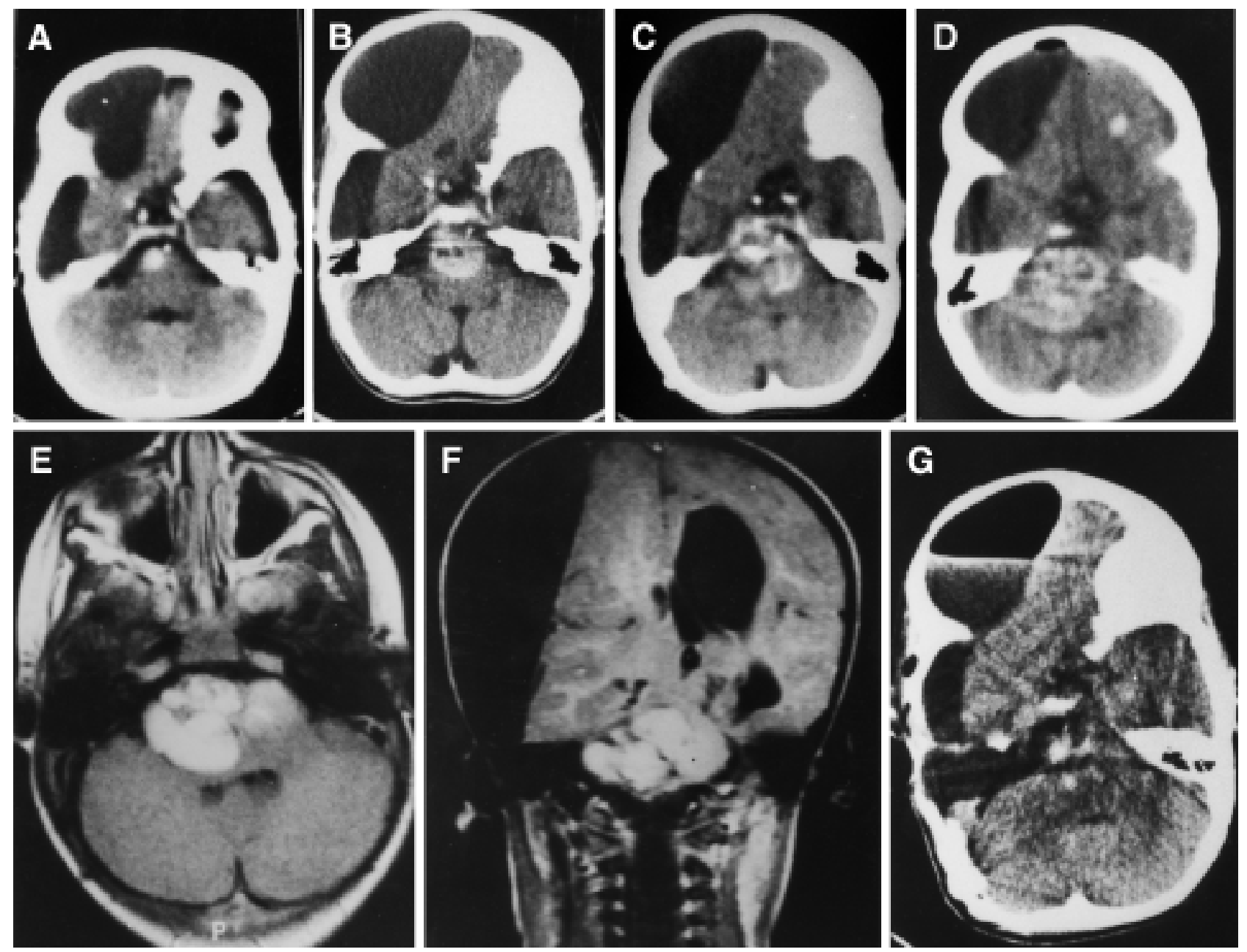

FIG. 1. Neuroimages obtained in a 3-year-old child who had previously undergone surgery for a right frontal cavernoma. A-D: Serial follow-up CT scans obtained after the addition of contrast material, revealing a brainstem cavernous angioma with progressive enlargement postsurgery (A, baseline; $\mathrm{B}, 4$ months; $\mathrm{C}, 1$ year; and D, 2 years). $\mathrm{E}$ and $\mathrm{F}$ : MR images obtained in axial (E) and coronal $(\mathrm{F})$ sequences after $\mathrm{Gd}$ administration, demonstrating a very large cavernoma occupying almost the whole brainstem and extending mainly on the right side. G: Postoperative CT scan of the brain after removal of the cavernomatous mass.

nent was expressed as a nuclear marker (number of positive nuclei from five random centers). A specimen of breast carcinoma was used as a positive control. The expression of bcl-2 was determined by using the monoclonal antibody anti-bcl-2 (Menarini, Florence, Italy; 1:100, 1-hour incubation in a humid chamber).

\section{Analysis of Immunostaining}

Immunopositivity for all growth factors was graded as 0 (absent), 1 (slight), 2 (intense but focal), and 3 (intense and diffuse). The immunopositivity for Ki-67 and bcl-2 was graded as 0 (absent), 1 (positivity $<5 \%$ ), 2 (positivity $5-10 \%$ ), and 3 (positivity $>10 \%$ ).

\section{Statistical Analysis}

Statistical analyses on contingent tables were conducted using commercially available software packages (SPSS version 13.0; SPSS, Inc., Chicago, IL; and Systat 11.0,
Richmond, CA). The rate of positivity of the growth factors and proliferative indices in the two groups of patients was compared using the Pearson chi-square test and the Fisher exact test (two-tailed). The level of significance was set at a probability value of 0.05 .

\section{Results}

The location of the cavernoma was frontal in 16 cases (37.2\%), temporal in $10(23.2 \%)$, parietal in nine $(20.9 \%)$, occipital in two $(4.7 \%)$, cerebellar in five $(11.7 \%)$, and pontine in one $(2.3 \%)$. The sizes ranged from 0.5 to $6 \mathrm{~cm}$ (mean $2 \mathrm{~cm}$ ). The clinical onset was characterized by epileptic seizures in 26 cases $(60.5 \%)$, focal neurological deficits in eight (18.6\%), and symptomatic extralesional hemorrhage in four $(9.3 \%)$, whereas in five patients $(11.6 \%)$ the cavernoma was discovered incidentally during neuroimaging studies for unrelated symptoms. 

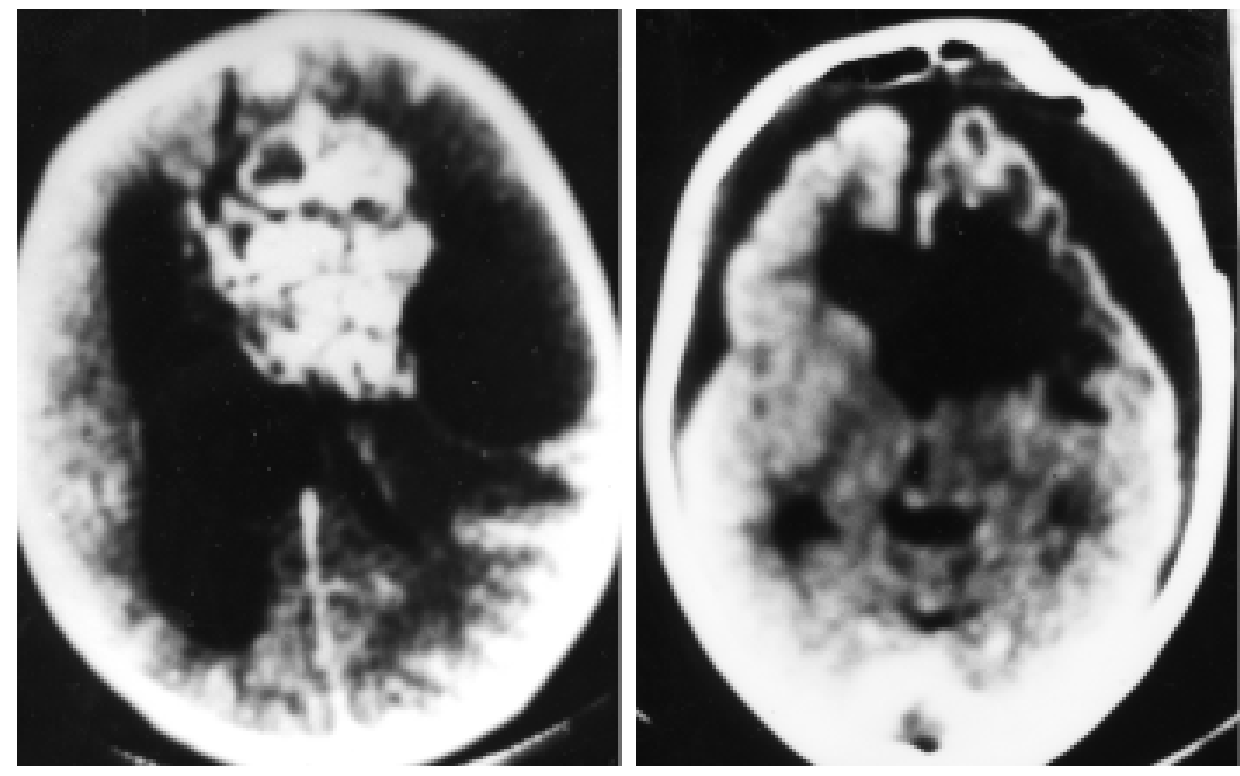

FIG. 2. Axial CT scans obtained in a 9-month-old infant who presented with generalized seizures and left hemiparesis. Left: Preoperative CT scan revealing a very large brain cavernoma consisting of a solid component in the median and right paramedian frontal regions and two large cysts in the right and left parietal regions. Right: Postoperative CT scan demonstrating complete removal of the cavernoma.

\section{Immunohistochemical Analysis}

The immunohistochemical studies of growth factors and proliferative indices provided the following results (see also Table 2). The VEGF expression was positive in the endothelium, subendothelium, and perivascular tissue in $36(83.7 \%)$ of 43 cases, manifesting mainly with slight or intense but focal positivity. On the other hand, perilesional brain parenchyma showed negative expression in all but two cases $(p<0.001)$. The cavernoma walls and perivascular tissue stained positively for tenascin in 36 (83.7\%) of 43 specimens, manifesting mainly as slight or intense but focal. In contrast, the perilesional brain parenchyma showed positive expression in only 10 cases $(23.3 \%)(\mathrm{p}<0.001)$.

The TGF $\beta$ expression was positive in the cavernoma walls and perivascular tissue in 25 cases $(58.1 \%)$ and in the perilesional brain parenchyma in $17(39.6 \%)(\mathrm{p}=$ $0.13)$.

The PDGF expression was positive in the vessel walls and perivascular tissue in 41 cases $(95.4 \%)$, mainly with intense (focal or diffuse) positivity, and perilesional brain parenchyma showed positive expression in 12 cases $(27.9 \%)(\mathrm{p}<0.001)$.

The expression of Ki-67 and bcl-2 in the cavernoma walls and perivascular tissue was negative in $35(81.4 \%)$ and $33(76.7 \%)$ cases, respectively. In seven and eight cases, respectively, Ki-67 and bcl-2 showed slight positivity of less than $5 \%$, whereas in only one case of multiple cavernomas was there intense positivity $(40 \%$ for Ki-67 and $15 \%$ for bcl-2). The expression of both markers in the perilesional brain parenchyma was negative in all but one case ( $p<0.001$ for both markers).

\section{Correlations of Clinical and Immunohistochemical Data}

Analysis of the clinical and immunohistochemical data of the two groups provided the following results (see also Tables 3 and 4).

Group I patients had a slightly higher mean age than those in Group II (35.4 compared with 25.2 years); all five children younger than 15 years were in Group II, which consisted of patients with more aggressive cavernomas. There were no significant differences in cavernous angioma locations in the two groups.

From the clinical viewpoint, in Group I there was a large prevalence of patients with epileptic seizures as the clinical presentation (24 [75\%] of 32 cases) and five $(15.6 \%)$ asymptomatic cases were diagnosed incidentally. On the other hand, Group II included five patients with neurological signs of mass effect, four with acute onset due to hemorrhage, and two with epileptic seizures. The differences in expression of growth factors and proliferative indices between the two groups are summarized in Tables 3 and 4.

The analysis of growth factor expression in the endothelium, subendothelium, and perivascular spaces of the cavernoma (Table 3 ) showed a high rate of positivity for VEGF, tenascin, and PDGF in both groups. The differences in the expression of these three growth factors were not significant $(p=0.656, p=0.656$, and $p=1$, respectively). The TGF $\beta$ was positive in all Group II cases and in only $14(43.7 \%)$ of 32 patients in Group I (p $=0.001$ ).

In cases with positive expression, the positivity was mainly slight or intense but focal in Group I, in which only five cases showed intense or diffuse positivity for PDGF, with one positive for VEGF and tenascin as well. On the other hand, positive cases in Group II mainly showed intense positivity (focal or diffuse), particularly for tenascin and PDGF.

The expression of proliferative indices was significantly different in the cavernoma tissue of the two groups, 

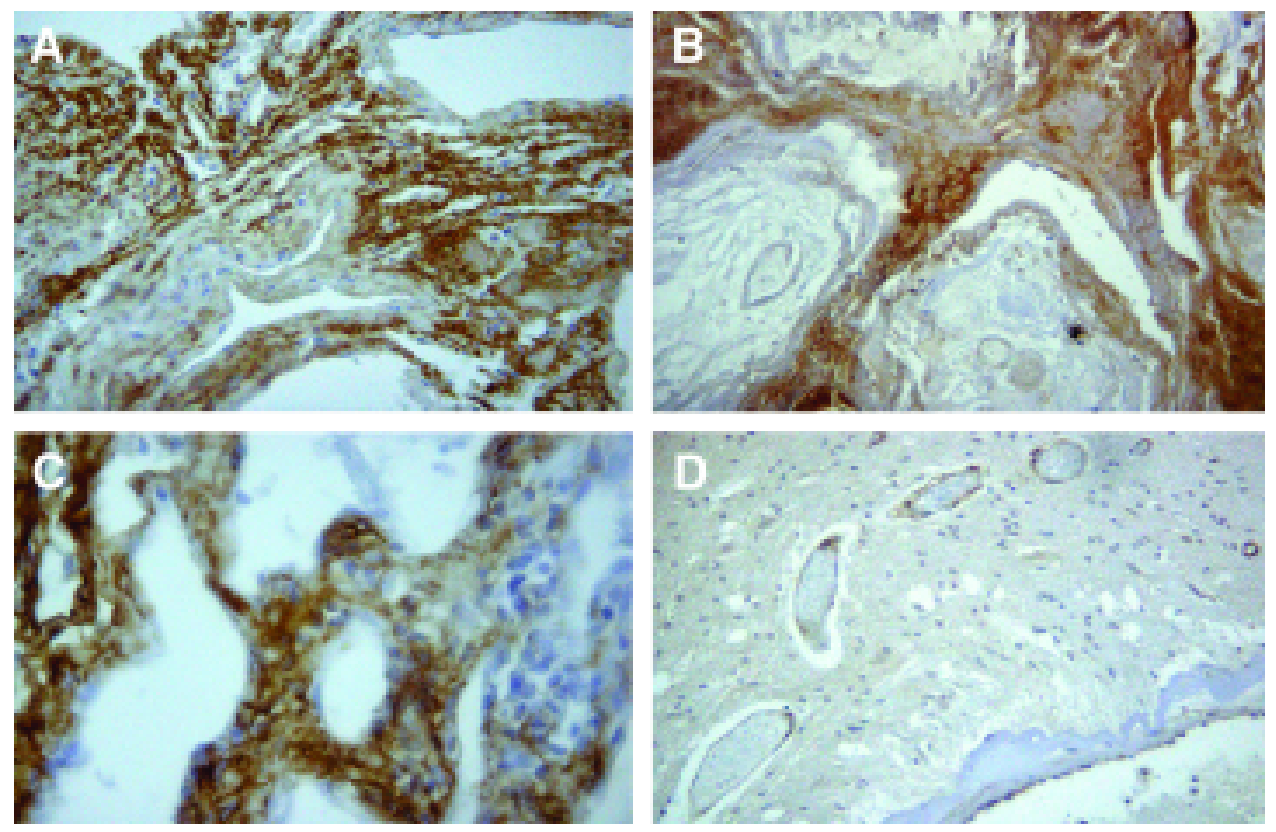

FIG. 3. Photomicrographs of the specimens prepared for immunohistochemical studies, showing positivity for VEGF (A), TGF $\beta$ (B), tenascin (C), and PDGF (D) in the surrounding brain parenchyma.

with eight $(72.7 \%)$ of 11 cases positive for both indices in Group II. In comparison, no cases in Group I were positive for Ki-67 and only two (6.25\%) of 32 cases were positive for bcl-2 ( $\mathrm{p}<0.001$ in both instances).

The analysis of growth factor expression in the perilesional brain parenchyma showed significant differences between the two groups (Table 4). Group II lesions, which included more aggressive cavernomas, showed a significantly higher rate of positivity for tenascin $(p=0.092)$, TGF $\beta(p=0.014)$, and PDGF ( $p=0.047)$ than the lesions in Group I, whereas the expression of VEGF was negative in more than $90 \%$ of the cases in both groups $(p=1)$. As expected, the expression of proliferative and apoptotic indices (Ki-67 and bcl-2) was also negative in all cases in both groups, except the slight (1\%) and nonsignificant positivity in one case in Group I.

\section{Discussion}

Brain cavernomas are malformative lesions occurring with an incidence of 0.3 to $0.6 \%$ in the general population. In the past, cavernous angiomas were considered to be biologically stable, nondeveloping lesions. ${ }^{2,8,21,27}$ However, investigators in several studies ${ }^{3,13,31,32,41}$ have described the enlargement and de novo formation of cavernous angiomas, $5,9,14,22,24,26,31,36$ thus confirming that these are dynamic and sometimes acquired lesions with potential clinical and biological progression..$^{15,22,31}$

Cavernous angiomas develop embryologically from malformed capillaries of the intraneural vascular territory that slowly enlarge and undergo obliteration and progressive fibrosis; gliosis of the interposed nervous tissue results in its complete destruction. Thus, the cavernoma develops in the final arrangement of the partially or com- pletely thrombosed vascular malformation without interposed nervous tissue.

Although the biological progression of cavernous angiomas is well recognized, the mechanisms of enlargement and growth are still being debated. Mechanisms of the malformation enlargement are well defined, and they include the following: 1) progressive ectasia of the vascular channels; 2) thrombosis of the contiguous vascular channels with fibrosis; 3) peripheral blood collections undergoing connective organization; and 4) formation of peripheral cysts resulting from internal hemorrhage. In some reports, ${ }^{20,34}$ however, investigators have suggested a role for proliferative mechanisms in the enlargement and growth of cavernous angiomas. These mechanisms include endothelial proliferation and neoangiogenesis, which are regulated by growth factors and ECM proteins.

Some recent immunohistochemical studies have shown expression of both proliferative indices and angiogenic and growth factors in brain cavernomas. ${ }^{6,29,37,43,45,46,48}$ Our study confirms that cavernomas express the main growth factors involved in angiogenesis.

A glycoprotein that mediates various phases of blood vessel development during vasculogenesis, VEGF was expressed in $83.7 \%$ of cavernous angiomas in our series and in 90 to $97 \%$ in two other studies ${ }^{18,48}$ whereas Sure, et al., ${ }^{42}$ found a lower rate of positivity (37.5-41\%). In agreement with the results of Kilic, et al., ${ }^{18}$ we found positive expression of VEGF, both in the vessel walls and in the interspaces between blood cavities.

The TGFs, a group of dimeric proteins that may hinder the growth of several cell types, regulate angiogenesis by stimulating the formation of blood vessels, and they also contribute to the formation and maintenance of the ECM. We have found TGF $\beta$ isomer expression in $54.2 \%$ of cavernous angiomas in our series. Two other studies ${ }^{18,48}$ report 
F. Maiuri, et al.

TABLE 2

Results of immunohistochemical studies for growth factors and proliferative indices in a series of 43 patients

\begin{tabular}{|c|c|c|c|c|c|c|c|c|}
\hline \multirow[b]{3}{*}{ Factor* } & \multicolumn{4}{|c|}{ Cavernoma } & \multicolumn{4}{|c|}{ Perilesional Brain Parenchyma } \\
\hline & \multirow{2}{*}{$\frac{\text { Negative }(\%)}{0}$} & \multicolumn{3}{|c|}{ Positive (\%) } & \multirow{2}{*}{$\frac{\text { Negative }(\%)}{0}$} & \multicolumn{3}{|c|}{ Positive (\%) } \\
\hline & & 1 & 2 & 3 & & 1 & 2 & 3 \\
\hline VEGF & $7(16.3)$ & $15(34.9)$ & $18(41.9)$ & $3(7)$ & $41(95.3)$ & $2(4.6)$ & $0(0)$ & $0(0)$ \\
\hline tenascin & $7(16.3)$ & $12(28)$ & $19(44.2)$ & $5(11.6)$ & $33(76.7)$ & $7(16.3)$ & $3(7)$ & $0(0)$ \\
\hline TGF $\beta$ & 18 (41.9) & $9(21)$ & $13(30.2)$ & $3(7)$ & $26(60.5)$ & 8 (18.6) & $6(14)$ & $3(7)$ \\
\hline PDGF & $2(4.6)$ & $11(25.5)$ & $20(46.5)$ & $10(23.3)$ & $31(72.1)$ & $8(18.6)$ & $1(2.3)$ & $3(7)$ \\
\hline Ki-67 & 35 (81.4) & 7 (16.3) & $0(0)$ & $1(2.3)$ & $42(97.7)$ & $1(2.3)$ & $0(0)$ & $0(0)$ \\
\hline bcl-2 & $33(76.7)$ & $8(18.6)$ & $1(2.3)$ & $1(2.3)$ & 42 (97.7) & $1(2.3)$ & $0(0)$ & $0(0)$ \\
\hline
\end{tabular}

* Immunopositivity scale for growth factors: $0=$ absent; $1=$ slight; $2=$ intense but focal; $3=$ intense and diffuse. Immunopositivity scale for Ki-67 and bcl-2: $0=$ absent; $1=$ positivity less than $5 \% ; 2=$ positivity 5 to $10 \% ; 3=$ positivity greater than $10 \%$.

positive expression of the $\alpha$ isomer in 97 to $100 \%$ of specimens, whereas there are no reports on the expression of the $\beta$ isomer of TGF in cavernous angiomas. This difference of expression between $\alpha$ and $\beta$ isomers deserves further investigation.

Tenascin, a glycoprotein of the ECM, is expressed during embryonic life and is absent in the normal brain. Evidence of this growth factor has been found in the vessels of anaplastic gliomas, and it is considered a marker of vascular proliferation for intracerebral growing lesions. We have found tenascin expression in $36(83.7 \%)$ of 43 cavernomas. Viale, et al., ${ }^{46}$ also found expression of tenascin-c in all 16 cavernomas in their series. The expression of tenascin in many cases of cavernous angiomas confirms the growth potential of such malformations.

A protein isolated from human platelets, PDGF stimulates the proliferation of connective and neuroglial cells and may play an important role in angiogenesis. The expression of PDGF has been described in some brain tumors, whereas there are no reports concerning this growth factor in cerebral vascular malformations. We found positive PDGF expression in $95.4 \%$ of cavernomas in the present series.

Therefore, this study confirms that cavernous angiomas strongly express some growth factors involved in the angiogenesis and formation of the ECM. Such factors are also expressed in some brain tumors, mainly in gliomas. This might indicate a growth potential for these vascular malformations.

The proliferative indices Ki-67 and PCNA were tested in four previous studies; the Ki-67 was always negative, $, 2,43,48$ whereas PCNA was positive in the nuclei of endothelial cells in $85 \%$ of cavernomas in two studies. ${ }^{29,42}$ Although both label proliferative cells, the significantly different expression of Ki-67 and PCNA may be explained by their different half-lives. ${ }^{43}$ We have found Ki67 positivity in the endothelium of eight $(18.6 \%)$ of 43 cavernomas in our series; all positive cases were lesions with more aggressive clinical behavior (Group II). This may explain the different results compared with other studies in which the clinical behavior of the examined cavernomas was not considered.

Apoptosis is known to be involved in the development and malignant progression of brain gliomas. It is regulated by the balance between antiapoptotic proteins (bcl-2 and bcl-XL) and proapoptotic proteins, which may activate the caspases. Only two reports have detailed the role of apoptosis in cavernous malformations. ${ }^{6,44}$ Takagi, et al., ${ }^{44}$ found caspase- 3 immunoreactivity in the endothelium, subendothelium, and intercavernous matrix in all five cavernous angiomas in their series. Cheng, et al., ${ }^{6}$ found increased expression of the antiapoptotic protein bcl-2 in 10 cavernomas. We have found bcl-2 positivity in 10 (23.3\%) of 43 specimens.

In this study, the reactive normal brain tissue adjacent to the cavernoma was found to express tenascin, TGF $\beta$, and PDGF, although in a rate significantly lower $(20-27.5 \%)$ than that found in the cavernoma tissue. The expression of these growth factors may reflect the gliotic reaction of the adjacent brain to repeated hemorrhages. Nevertheless, VEGF was not expressed in the reactive brain tissue, as was reported by Kilic, et al., ${ }^{18}$ probably because of the sparse angiogenic reaction in the brain adjacent to the cavernomas.

In several reports, researchers have investigated the natural history of cerebral cavernomas, both the sporadic and familial forms. ${ }^{2,7,8,15,19,21-23,30}$ They confirm that some cases show a more aggressive course. Factors associated with clinical and radiological progression include age, familial occurrence, large size with mass effect, documented growth, de novo appearance, and significant hemorrhage. Evolutive cavernomas are more frequent in infancy, mainly in the 1st year of life. ${ }^{11}$

Familial forms represent 25 to $54 \%$ of all cavernomas; ${ }^{12}$ they are characterized by genetic transmission as an auto-

TABLE 3

Statistical analysis of the growth factors and proliferative indices assessed in cavernoma tissue resected in two groups of patients*

\begin{tabular}{lccc}
\hline \hline Factor & Group I & Group II & p Value \\
\hline VEGF & $26(81.25)$ & $10(90.9)$ & 0.656 \\
tenascin & $26(81.25)$ & $10(90.9)$ & 0.656 \\
TGF $\beta$ & $14(43.75)$ & $11(100)$ & 0.001 \\
PDGF & $30(93.75)$ & $11(100)$ & 1 \\
Ki-67 & $0(0)$ & $8(72.7)$ & $<0.001$ \\
bcl-2 & $2(6.25)$ & $8(72.7)$ & $<0.001$
\end{tabular}

* Group I included 32 patients with indolent or stable lesions; Group II included 11 patients with larger lesions that exhibited aggressive behavior. Numbers in parentheses represent percentages. 
Growth factors in progression of cavernous angiomas

TABLE 4

Statistical analysis of the growth factors and proliferative indices assessed in perilesional brain parenchyma resected in two groups of patients*

\begin{tabular}{llll}
\hline \hline Factor & Group I & Group II & p Value \\
\hline VEGF & $2(6.25)$ & $1(9)$ & 1 \\
tenascin & $5(15.6)$ & $5(45.5)$ & 0.092 \\
TGF & $9(28.1)$ & $8(72.7)$ & 0.014 \\
PDGF & $6(18.8)$ & $6(54.5)$ & 0.047 \\
Ki-67 & $1(3.13)$ & $0(0)$ & 1 \\
bcl-2 & $1(3.13)$ & $0(0)$ & 1 \\
\hline
\end{tabular}

* See Table 3 for description of Groups I and II. Numbers in parentheses represent percentages.

somal-dominant trait, more aggressive behavior, and a higher rate of multiple localizations (66 compared with $10 \%$ ) than the sporadic cases. ${ }^{25,23}$ In a series of 40 familial cerebral cavernomas, Labauge, et al., ${ }^{22,23}$ found changes in size in $70 \%$, bleeding in $35 \%$, and de novo lesions in $27.5 \%$. However, factors related to this more aggressive course have not been defined.

Very large cavernomas (up to 6-7 $\mathrm{cm}$ in diameter) are unusual and are associated with symptoms and signs of mass effect; in some cases a growth of the mass has been documented on serial CT and MR imaging studies. ${ }^{4,13,29,31 \text {, }}$ ${ }^{32,38}$ Progressive thrombosis of the vascular channels, conspicuous or recurrent intralesional hemorrhages, and formation of both intralesional and extralesional cysts account for the large or increasing size of some cavernomas.

De novo formation of cavernomas has been reported in both sporadic and familial cases, $1,9,16,22,26,31,36,39,40$ sometimes after radiation therapy ${ }^{10,14,24}$ or immunosuppressive treatment. ${ }^{5}$ Whether the de novo appearance may be due to the growth of small lesions that were previously undetectable, or neuroimaging, or to truly de novo, acquired lesions caused by pathological angiogenesis is still unclear. It is likely that in patients who have previously undergone radiation therapy, the treatments may stimulate endothelial proliferation and angiogenesis, thus leading to cavernoma formation.

Our study was conducted to investigate whether the familial occurrence and the more aggressive clinical course of some brain cavernomas may be correlated with differences in the expression of growth factors and proliferative indices. Thus, we have compared the immunohistochemical data found in more aggressive cavernomas with larger $(>2 \mathrm{~cm})$ size, mass effect, documented growth, and significant extralesional hemorrhage with those of stable and sporadic cavernomas that have no symptoms or only occasional seizures. No previous reports have investigated this correlation. Only Jung, et al., ${ }^{17}$ found increased VEGF expression in a case of multiple cavernomas with a progressive course.

Analysis of the growth factors in the cavernoma tissue (vessel walls and perivascular tissue) shows a higher rate of positive TGF $\beta$ expression in the group of more aggressive lesions, whereas the expression of VEGF, PDGF, and tenascin showed no differences between the two groups. On the other hand, more aggressive cavernomas exhibit a significantly higher expression of TGF $\beta$, PDGF, and tenascin in the perilesional reactive brain parenchyma, whereas VEGF expression is always absent in both stable and aggressive lesions.

The significantly higher expression of all growth factors except the VEGF in the perilesional brain parenchyma in cases of cavernomas with documented growth, significant perilesional hemorrhage, and large size $(\leq 6 \mathrm{~cm})$ is a very interesting finding in our opinion. The enlargement of the cavernomatous matrix may be due to reendothelialization within the small hematoma ${ }^{28,39}$ or to stimulation of connective tissue proliferation by hemorrhage and neovascularization. ${ }^{25}$ In all these cases, the expression in the perilesional brain parenchyma of TGF $\beta$, PDGF, and tenascin, which are all involved in the formation of the ECM, may reflect glial proliferation in the surrounding brain. This suggests that the perilesional brain parenchyma surrounding more aggressive cavernomas might be predisposed to receive the growing cavernomatous matrix.

The absence of VEGF expression in the perilesional brain of all but two cavernomas in our series, which included both stable and aggressive lesions, may indicate the absence of true angiogenesis in the brain vessels. The VEGF is an important growth factor involved in the progression of brain gliomas, where it is correlated with the tumor grade and vascularization. On the other hand, its role in the progression of cavernous angiomas is controversial.

Jung, et al., ${ }^{17}$ found a significant increase in VEGF expression in the pathological specimens as well as increased concentration of this factor in the serum of a patient with multiple growing and de novo cavernomas that were causing multiple intracerebral microhemorrhages. These authors speculated that the endothelial proliferation induced by VEGF is an important element in the development of brain cavernomas. The data in our study do not support this statement. In fact, we found no statistically significant difference in the VEGF expression, either in the cavernomatous tissue or in the perilesional brain parenchyma, between stable and more aggressive forms. This suggests that the significant increase of VEGF expression in some cases may reflect a nonspecific pathological reaction to intracranial hemorrhage rather than a true endothelial proliferation.

The results of proliferative indices are another interesting finding in our study. The expression of both the Ki-67 labeling index and bcl-2 was absent in the group of stable lesions, and it was positive in eight $(72.7 \%)$ of the 11 more aggressive cavernomas. This may indicate a tendency in larger cavernomas toward growth accompanied by clinical progression.

\section{Conclusions}

The expression of some growth factors (such as VEGF, TGF $\beta$, PDGF, and tenascin) in brain cavernous malformations shows that they are dynamic lesions with growth potential, even when they are observed as stable lesions with an indolent course.

More evolutive cavernomas are associated with a significantly higher expression of Ki-67 and bcl-2, as in other proliferative lesions.

The perilesional brain parenchyma surrounding caver- 
nomas that exhibit more aggressive clinical behavior expresses TGF $\beta$, PDGF, and tenascin at a significantly higher rate than is found in stable and more indolent lesions. This may indicate that the perilesional nervous and glial tissue may be predisposed and recruited for further growth and progression of the cavernoma, ultimately adding new blood cavities for the enlargement of these lesions.

\section{References}

1. Agazzi S, Maeder P, Villemure JG, Regli L: De novo formation and growth of a sporadic cerebral cavernous malformation: implications for management in an asymptomatic patient. Cerebrovasc Dis 16:432-435, 2003

2. Aiba T, Tanaka R, Koike T, Kameyama S, Takeda N, Komata T: Natural history of intracranial cavernous malformations. J Neurosurg 83:56-59, 1995

3. Bertalanffy H, Benes L, Miyazawa T, Alberti O, Siegel AM, Sure U: Cerebral cavernomas in the adult. Review of the literature and analysis of 72 surgically treated patients. Neurosurg Rev 25:1-55, 2002

4. Bertalanffy H, Gilsbach JM, Eggert HR, Seeger W: Microsurgery of deep-seated cavernous angiomas: report of 26 cases. Acta Neurochir 108:91-99, 1991

5. Brunken M, Sagehorn S, Leppien A, Muller-Jensen A, Halves $\mathrm{E}$ : [De novo formation of a cavernoma in association with a preformed venous malformation during immunosuppressive treatment.] Zentralbl Neurochir 60:81-85, 1999 (Ger)

6. Cheng L, Liang J, Tang S: [The study on the role of apoptosis suppressive gene bcl-2 in the pathogenesis of hemangioma.] Zhonghua Zheng Xing Shao Shang Wai Ke Za Zhi 15: 35-36, 1999 (Chi)

7. Clatterbuck RE, Moriarity JL, Elmaci I, Lee RR, Breiter SN, Rigamonti D: Dynamic nature of cavernous malformations: a prospective magnetic resonance imaging study with volumetric analysis. J Neurosurg 93:981-986, 2000

8. Del Curling O Jr, Kelly DL Jr, Elster AD, Craven TE: An analysis of the natural history of cavernous angiomas. J Neurosurg 75:702-708, 1991

9. Detwiler PW, Porter RW, Zabramski JM, Spetzler RF: De novo formation of a central nervous system cavernous malformation: implications for predicting risk of hemorrhage. Case report and review of the literature. J Neurosurg 87:629-632, 1997

10. Detwiler PW, Porter RW, Zabramski JM, Spetzler RF: Radiation-induced cavernous malformation. J Neurosurg 89: $167-169,1998$

11. Gangemi M, Longatti P, Maiuri F, Cinalli G, Carteri A: Cerebral cavernous angiomas in the first year of life. Neurosurgery 25:465-469, 1989

12. Gangemi M, Maiuri F, Donati P, Cinalli G, De Caro M, Sigona L: Familial cerebral cavernous angiomas. Neurol Res 12: 131-136, 1990

13. Gangemi M, Maiuri F, Donati PA, Sigona L: Rapid growth of a brain-stem cavernous angioma. Acta Neurol (Napoli) 15: 132-137, 1993

14. Heckl S, Aschoff A, Kunze S: Radiation-induced cavernous hemangiomas of the brain. A late effect predominantly in children. Cancer 94:3285-3291, 2002

15. Houtteville JP: Brain cavernoma: a dynamic lesion. Surg Neurol 48:610-614, 1997

16. Jain KK, Robertson E: Recurrence of an excised cavernous hemangioma in the opposite cerebral hemisphere. Case report. J Neurosurg 33:453-456, 1970

17. Jung KH, Chu K, Jeong SW, Park HK, Bae HJ, Yoon BW: Cerebral cavernous malformations with dynamic and progressive course. Correlation study with vascular endothelial growth factor. Arch Neurol 60:1613-1618, 2003

18. Kilic T, Pamir MN, Kullu S, Eren F, Ozek MM, Black PM:
Expression of structural proteins and angiogenic factors in cerebrovascular anomalies. Neurosurgery 46:1179-1192, 2000

19. Kim DS, Park YG, Choi JU, Chung SS, Lee KC: An analysis of the natural history of cavernous malformations. Surg Neurol 48:9-17, 1997

20. Klagsbrun M, D'Amore PA: Regulators of angiogenesis. Annu Rev Physiol 53:217-239, 1991

21. Kondziolka D, Lunsford LD, Kestle JR: The natural history of cerebral cavernous malformations. J Neurosurg 83:820-824, 1995

22. Labauge P, Brunereau L, Laberge S, Houtteville JP: Prospective follow-up of 33 asymptomatic patients with familial cerebral cavernous malformations. Neurology 57:1825-1828, 2001

23. Labauge P, Brunereau L, Levy C, Laberge S, Houtteville JP: The natural history of familial cerebral cavernomas: a retrospective MRI study of 40 patients. Neuroradiology 42: 327-332, 2000

24. Larson JJ, Ball WS, Bove KE, Crone KR, Tew JM Jr: Formation of intracerebral cavernous malformations after radiation treatment for central nervous system neoplasia in children. J Neurosurg 88:51-56, 1998

25. LeDoux MS, Aronin PA, Odrezin GT: Surgically treated cavernous angiomas of the brain stem: report of two cases and review of the literature. Surg Neurol 35:395-399, 1991

26. Massa-Micon B, Luparello V, Bergui M, Pagni CA: De novo cavernoma case report and review of literature. Surg Neurol 53:484-487, 2000

27. Moriarity JL, Wetzel M, Clatterbuck RE, Javedan S, Sheppard JM, Hoenig-Rigamonti K, et al: The natural history of cavernous malformations: a prospective study of 68 patients. Neurosurgery 44:1166-1173, 1999

28. Notelet L: Les cavernomes cérébraux: étude des méchanismes d'acroissement et étude génétique d'une forme familiale, in Caen: Université de Caen, 1996, pp 1-163 (unpublished thesis)

29. Notelet L, Houtteville JP, Khoury S, Lechevalier B, Chapon F: Proliferating cell nuclear antigen (PCNA) in cerebral cavernomas: an immunocytochemical study of 42 cases. Surg Neurol 47:364-370, 1997

30. Porter PJ, Willinsky RA, Harper W, Wallace MC: Cerebral cavernous malformations: natural history and prognosis after clinical deterioration with or without hemorrhage. J Neurosurg 87: 190-197, 1997

31. Pozzati E, Acciarri N, Tognetti F, Marliani F, Giangaspero F: Growth, subsequent bleeding, and de novo appearance of cerebral cavernous angiomas. Neurosurgery 38:662-670, 1996

32. Pozzati E, Giuliani G, Nuzzo G, Poppi M: The growth of cerebral cavernous angiomas. Neurosurgery 25:92-97, 1989

33. Rigamonti D, Hadley MN, Drayer BP, Johnson PC, HoenigRigamonti K, Knight JT, et al: Cerebral cavernous malformations. Incidence and familial occurrence. N Engl J Med 319: 343-347, 1988

34. Risau W: Mechanisms of angiogenesis. Nature 386:671-674, 1997

35. Robinson JR, Awad IA, Little JR: Natural history of the cavernous angioma. J Neurosurg 75:709-714, 1991

36. Rosahl SK, Vorkapic P, Eghbal R, Ostertag H, Samii M: Ossified and de novo cavernous malformations in the same patient. Clin Neurol Neurosurg 100:138-143, 1998

37. Rothbart D, Awad IA, Lee J, Kim J, Harbaugh R, Criscuolo GR: Expression of angiogenic factors and structural proteins in central nervous system vascular malformations. Neurosurgery 38:915-925, 1996

38. Savoiardo M, Passerini A: CT, angiography, and RN scans in intracranial cavernous hemangiomas. Neuroradiology 16: 256-260, 1978

39. Scott RM, Barnes P, Kupsky W, Adelman LS: Cavernous angiomas of the central nervous system in children. J Neurosurg 76:38-46, 1992 


\section{Growth factors in progression of cavernous angiomas}

40. Siegel AM: Familial cavernous angioma: an unknown, known disease. Acta Neurol Scand 98:369-371, 1998

41. Stacey RJ, Ashkan K, Edwards JMR: Rapid growth in a cavernoma. Br J Neurosurg 14:585-588, 2000

42. Sure U, Butz N, Schlegel J, Siegel AM, Wakat JP, Mennel HD, et al: Endothelial proliferation, neoangiogenesis, and potential de novo generation of cerebrovascular malformations. J Neurosurg 94:972-977, 2001

43. Sure U, Freman S, Bozinov O, Benes L, Siegel AM, Bertalanffy $\mathrm{H}$ : Biological activity of adult cavernous malformations: a study of 56 patients. J Neurosurg 102:342-347, 2005

44. Takagi Y, Hattori I, Nozaki K, Ishikawa M, Hashimoto N: DNA fragmentation in central nervous system vascular malformations. Acta Neurochir (Wien) 142:987-994, 2000

45. Uranishi R, Baev NI, Ng PY, Kim JH, Awad IA: Expression of endothelial cell angiogenesis receptors in human cerebrovascular malformations. Neurosurgery 48:359-368, 2001

46. Viale GL, Castellani P, Dorcaratto A, Pau A, Sehrbundt E, Siri A, et al: Occurrence of a glioblastoma-associated tenascin-C isoform in cerebral cavernomas and neighboring vessels. Neurosurgery 50:838-842, 2002

47. Zabramski JM, Wascher TM, Spetzler RF, Johnson B, Golfinos J, Drayer BP, et al: The natural history of familial cavernous malformations: results of an ongoing study. J Neurosurg 80: 422-432, 1994

48. Zhao Y, Mao Y, Zhou LF, Zhang YL: [Immunohistochemical study on central nervous system cavernous hemangiomas.] Zhonghua Yi Xue Za Zhi 83:544-547, 2003 (Chi)

Manuscript received February 6, 2006.

Accepted in final form May 30, 2006.

Address reprint requests to: Paolo Cappabianca, M.D., Dipartimiento di Scienze Neurologiche, Divisione di Neurochirurgia, Università degli Studi di Napoli Federico II, School of Medicine, Via S. Pansini 5, 80131 Napoli, Italy. email: paolo.cappabianca@unina.it. 